\title{
Pointwise and Uniform Power Series Convergence
}

\author{
C. D'Apice, G. Gargiulo, and R. Manzo \\ University of Salerno, Department of Information Engineering and Applied Mathematics, \\ via Ponte don Melillo,84084 Fisciano (SA), Italy \\ \{dapice, gargiulo, manzo\} @diima.unisa.it
}

\begin{abstract}
Since the introduction of CAS (Computer Algebra Systems), educators are experimenting new ways of teaching with the aim to enhance many aspects of the learning mathematics. In particular, visualization can aid the understanding of concepts. The graphical potentialities of many CAS, in fact, allow students to discover concepts, relationships, rules, so as to construct their knowledge for themselves. The aim of this work is to present a Mathematica notebook that enables students to use visualization skills to better grasp the mathematical concepts of the pointwise and uniform convergence of power series.
\end{abstract}

\section{Visualization in Mathematics Teaching}

Visualization, as a technique for teaching mathematics, has become importante specially in those countries where graphics calculators are widely used. Mathematical concepts, ideas and methods have a great wealth of visual relationships, and their use and manipulation is clearly very beneficial from the point of view of their presentation.

Visualization means illustration of an object, fact, process, concept, and its result can be graphic, numeric, or algebraic. Today the term is mostly used for graphic illustrations. Visualization tools can be used in an interpretative and expressive way (Gordin, Edelson and Gomez, 1996).

Interpretative tools can support learners in extracting meaning from the information being visualized, in clarifying text and abstract concepts making them more comprehensible. Expressive visualization can be used to visually convey meaning in order to communicate a set of beliefs.

When using a calculator as a visualization tool the immediate feedback is of central importance. The illustration of concepts helps students to understand them, to associate them with something that is not abstract and the visual association aids to memorize them. Student become able to "see" the meaning of abstract patterns, interacting visually with mathematics.

Mathematica, being a powerful tool for computing, programming, data analysis and visualization of information, is able to catch students' interests, to challenge them and to make learning more interesting.

The use of Mathematica notebooks, as a support to traditional lessons, can help students in understanding some concepts, since they are personally involved in experimentation and discovery. We have introduced them in the traditional curriculum for some years, within a research addressed to the development of innovative ways of mathematics teaching using Mathematica. 
Here we describe a notebook that can offer useful visualization of the concepts of pointwise and uniform convergence of power series.

\section{Pointwise and Uniform Convergence of Power Series}

The notebook, we are going to illustrate, has been realized with the aim to aid students to reach a deeper knowledge of the concepts of pointwise and uniform convergence of power series. Students used it after traditional lessons on the above contents in order to explore the meaning of convergence.

To introduce the convergence of power series, we recall the following definitions.

Definition 1. Let I be a subset of $R,\left\{f_{n}\right\}, f_{n}: I \rightarrow \mathbb{R}$, be a sequence of functions, and let $f: I \rightarrow \mathbb{R}$ be another function. We say that $f_{n} \rightarrow f$

- pointwise on I if $\forall x \in I$ and $\forall \varepsilon>0 \exists N(x, \in)$ such that $n>N \Rightarrow\left|f_{n}(x)-f(x)\right|<\epsilon$ - uniformly on I if $\forall \in>0 \exists N(\in)$ such that $n>N \Rightarrow\left|f_{n}(x)-f(x)\right|<\in, \forall x \in I$.

The graphics in Figure 1 illustrates how, for sufficiently large n, the n-th function $f_{n}(x)=x e^{-n x}$ lies completely below any given level (in the form $\frac{1}{e n}$ ). In this case, for simplicity, the (uniform) limit is identically zero, so that close to the limit simply means small. In the case of (pointwise but) non uniform convergence, the n-th function stays below any given level, but not globally: the maximum is essentially the same for all functions (here $f_{n}(x)=n x e^{-n x}$ ).
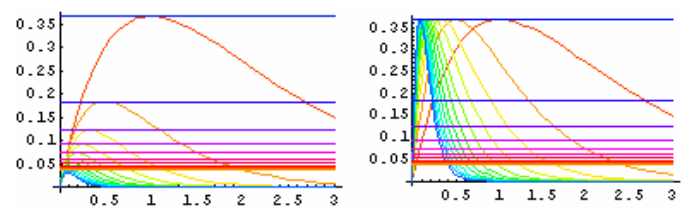

Fig. 1. On the left $f_{n}(x)=x e^{-n x}$, on the right $f_{n}(x)=n x e^{-n x}$

Definition 2. The series $s(x)=\sum_{k=1}^{\infty} u_{k}(x)$ converges pointwise to the function $s$ if the sequence $s_{n}=\sum_{k=1}^{n} u_{k}(x)$ converges pointwise to the function $s$.

Definition 3. The series $s(x)=\sum_{k=1}^{n} u_{k}(x)$ converges uniformly to the function $s$ if the sequence $s_{n}(x)$ converges uniformly to the function $s$.

Let us go to visually study the convergence of some power series. 


\subsection{Series $\sum_{k=0}^{\infty} z^{k}$}

Let us consider the geometric series $\sum_{k=0}^{\infty} z^{k}$ which converges within the circle of convergence $|z|<1$ and diverges outside $\sum_{k=0}^{\infty} z^{k}=\frac{1}{1-z}$. Let us analyze the behaviour of the partial sums of the geometric series in the complex plane using the following function:

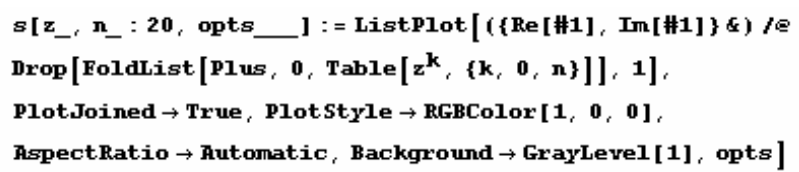

Visualize the behavior of the first 40 partial sums of the series in a point, $\frac{3}{5}+\frac{4 i}{5}$, on the boundary of the convergence circle.

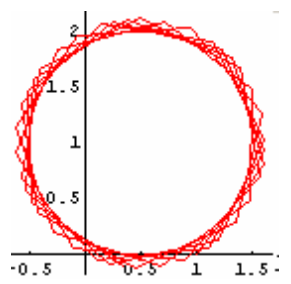

Fig. 2. $s\left[\frac{3}{5}+\frac{4 i}{5}, 40\right]$

As we can see the geometric series does not converge, since the points representing the partial sums do not "head" towards a definite point. Let us consider (Fig. 3) the behaviour of the first 200 partial sums in a point, $0.99\left(\frac{3}{5}+\frac{4 i}{5}\right)$, inside the circle of convergence. The geometric series converges but since the point is near the circle the

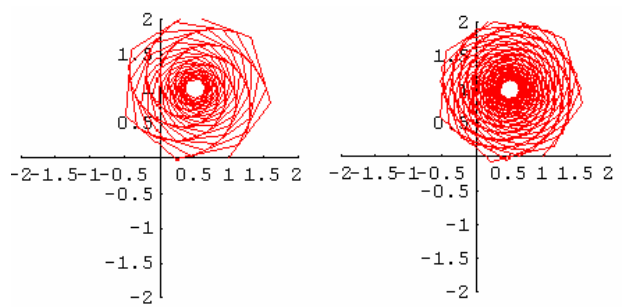

Fig. 3. On the left $s\left[0.99\left(\frac{3}{5}+\frac{4 i}{5}\right), 200\right]$, on the right $s\left[0.995\left(\frac{3}{5}+\frac{4 i}{5}\right), 20\right]$ 
convergence is slow: the sums slowly spiral towards their limit. Let us consider (Fig. 3) a point, $0.995\left(\frac{3}{5}+\frac{4 i}{5}\right)$,nearer the circle: the series converges but the convergence is even slower than before.

In a point, $0.9\left(\frac{3}{5}+\frac{4 i}{5}\right)$, far from the boundary of the convergence circle, the convergence is faster as we can see in the following graphics.

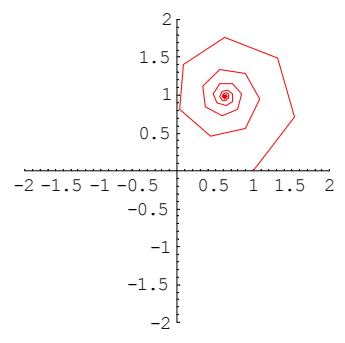

Fig. 4. $s\left[0.9\left(\frac{3}{5}+\frac{4 i}{5}\right), 200\right]$

\subsection{Series $\sum_{k=1}^{\infty} \frac{z^{k}}{k}$}

Let us analyze the convergence of the series $\sum_{k=1}^{\infty} \frac{z^{k}}{k}=-\log [1-z]$. First, we consider the same point, $\frac{3}{5}+\frac{4 i}{5}$, as before, on the boundary of the circle of convergence (Fig. 5). This time, the series converges (slowly) also in this point. Then let us analyze the behaviour in a point, $0.99\left(\frac{3}{5}+\frac{4 i}{5}\right)$, inside the circle (Fig. 5).

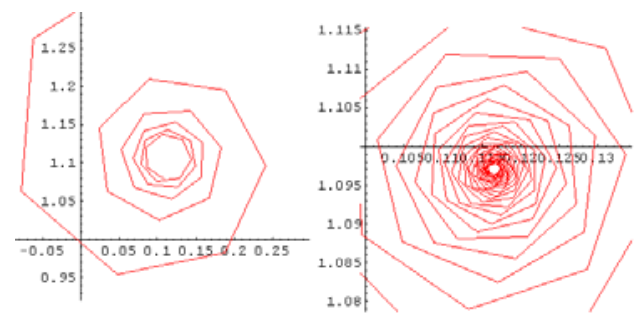

Fig. 5. On the left $s\left[\left(\frac{3}{5}+\frac{4 i}{5}\right), 40\right]$, on the right $s\left[0.99\left(\frac{3}{5}+\frac{4 i}{5}\right), 20\right]$ 
We "zoom out" the graphics and we consider a point still closer to the boundary and a point farther from the boundary (Fig. 6).
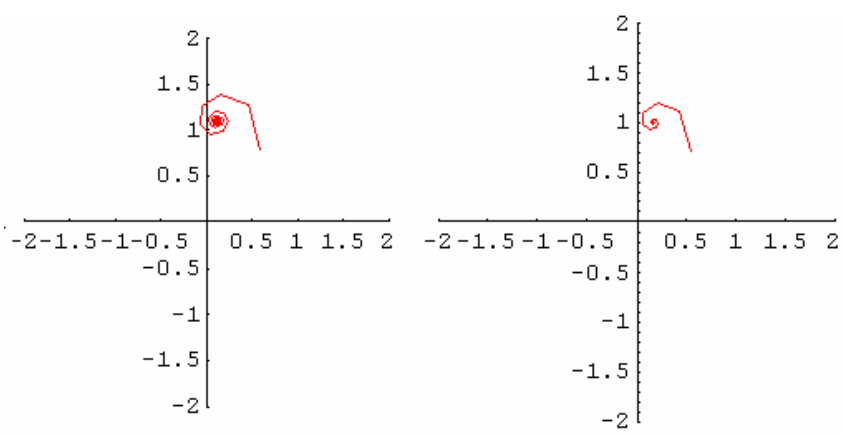

Fig. 6. On the left $s\left[0.995\left(\frac{3}{5}+\frac{4 i}{5}\right), 400\right]$, on the right $s\left[0.9\left(\frac{3}{5}+\frac{4 i}{5}\right), 400\right]$

\section{Uniform Convergence}

Let us define a general function that allows to study the behaviour of the partial sums of a power series in a chosen point.

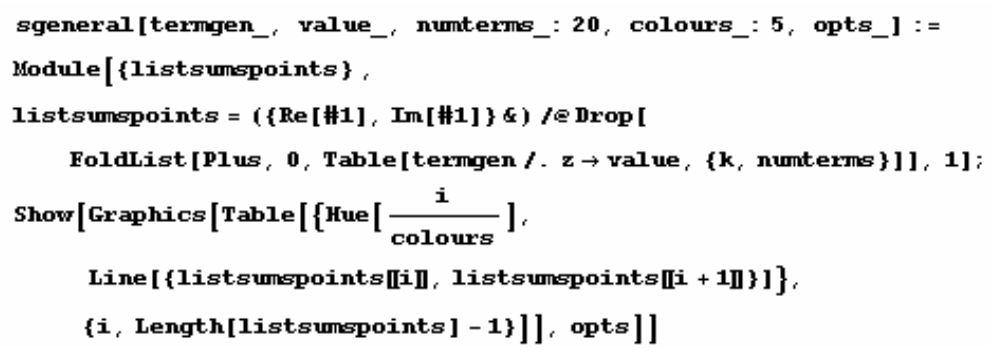

Let us try to understand the meaning of uniform convergence, observing the behaviour of the partial sums in different points, in polar coordinates (i.e. we pick up points in given directions and on several circles around the origin).

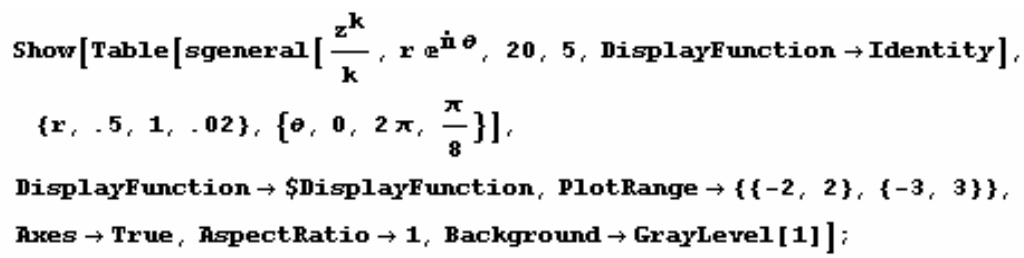

As we can see the convergence is faster in the points far from the boundary of the convergence circle, and becomes slow near the boundary. 


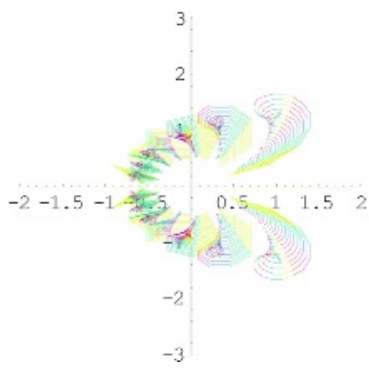

Fig. 7. The series with general term $\frac{z^{k}}{k}$

The series does not converge uniformly, in fact the convergence "rates" depends in an essential manner on the point.

With different general terms, $\frac{z^{k}}{k \log [k+1]}$ and $\frac{z^{k}}{\sqrt{k}}$ (Fig. 7), we see the same general pattern as before, but the convergence rate is a trifle higher (spirals are someway smoother).

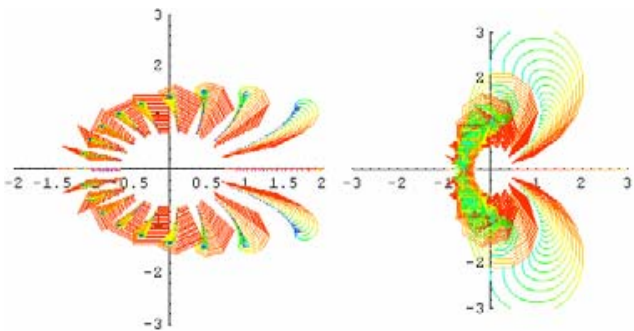

Fig. 8. The series with general term $\frac{z^{k}}{k \log [k+1]}$ on the left and $\frac{z^{k}}{\sqrt{k}}$ on the right

The geometric series again around the point 0.95 and still closer to the boundary around the point 0.95 (Fig. 8).
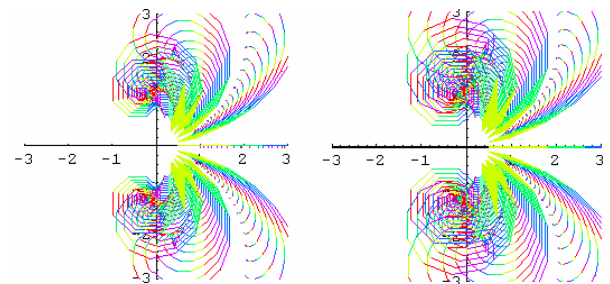

Fig. 9. The geometric series 
Below are shown two examples of uniform convergence, the series with general term $\frac{z^{k}}{k^{2}}$ and $\frac{z^{k}}{k^{4}}$ (Fig. 9).

We "see" that the spirals have the same general ("uniform") structure. The second series is still "more uniform" example: as Euler would have said, an "augmented convergence".
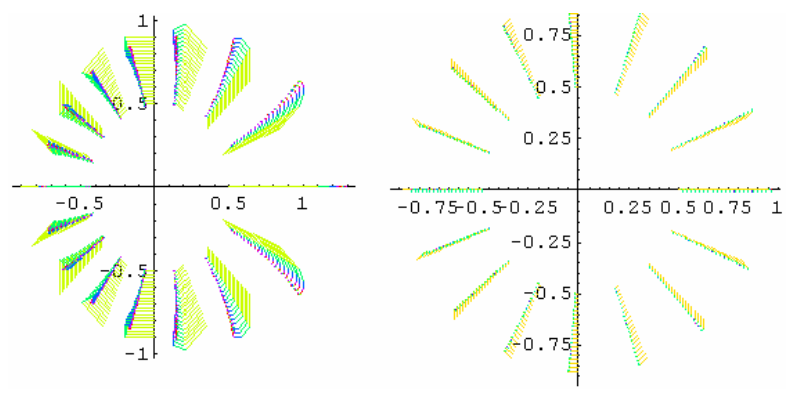

Fig. 10. The partial sums of series with general term $\frac{z^{k}}{k^{2}}$ on the left and $\frac{z^{k}}{k^{4}}$ on the right

The logarithmic series around its singular point and "very close" to its boundary singular point $z=1$.

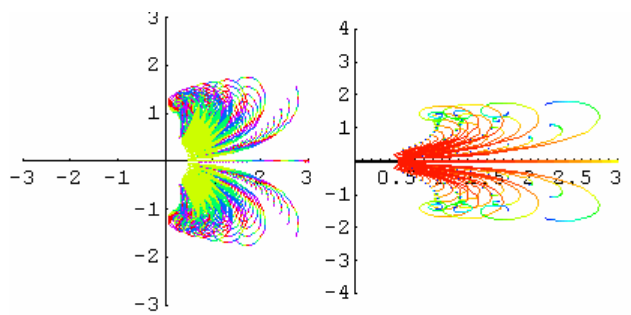

Fig. 11. The logarithmic series

\section{Conclusions}

The realization of this notebook encourages the use of computer graphical capabilities in teaching mathematics contents in University courses. The results of their use can be seen as a step towards the introduction of innovative modes in the process of teaching and learning. As a result of the experimentation students have enhanced their understanding of the illustrated concepts, learning in a more enjoyable way. 


\section{References}

1. G. Albano, C. D'Apice, G. Gargiulo: Visualisation of four dimensional curves, Electronic Proceedings ICTMT4 (International Conference on Technology in Mathematics Teaching), Plymouth, 1999;

2. Albano, G., Cavallone, A., D'Apice, C., Gargiulo, G.: Mathematica and didactical innovation: a quadric use case, Electronic Proceedings of IMACS-ACA Conferences on Applications of Computer Algebra, Madrid, (1999);

3. Cavallone, A., D'Apice, C., Marsella, M., Salerno, S.: A didactical laboratory: image filtering, Electronic Proceedings of IMACS-ACA Conferences on Applications of Computer Algebra, Madrid, (1999);

4. D'Apice, C., De Simone, T., Manzo, R., Tibullo, V.: MOSFET: a Virtual Laboratory with Mathematica, Proceedings of ICTMT6 Volos, Greece, (2003), 252-257;

5. D'Apice, C., Manzo R., Tibullo, V.: Enhancing Mathematical Teaching-Learning Process by Mathematica, Proceedings of the International Mathematica Symposium 2003, London, (2003), 137-144.

6. Giusti, E. : Analisi Matematica II, Bollati Boringhieri, Torino, 1994.

7. Miranda, C. ; Lezioni di Analisi Matematica, parte seconda, Liguori Editore, Napoli, 1985. 